\title{
Study of $f_{0}$ Decays into Four Neutral Pions
}

\section{The CRYSTAL BARREL collaboration}

\author{
A. Abele ${ }^{8}$, J. Adomeit ${ }^{7}$, C. Amsler ${ }^{14}$, C.A. Baker ${ }^{5}$, B.M. Barnett ${ }^{3}$ a, C.J. Batty ${ }^{5}$, M. Benayoun ${ }^{11}$, S. Bischoff ${ }^{8}$, \\ P. Blüm ${ }^{8}$, K. Braune ${ }^{10}$, T. Case ${ }^{1}$, V. Credé ${ }^{3}$, K.M. Crowe ${ }^{1}$, T. Degener ${ }^{2}$, M. Doser ${ }^{6}$, W. Dünnweber ${ }^{10}$, \\ D. Engelhardt ${ }^{8}$, M.A. Faessler ${ }^{10}$, P. Giarritta ${ }^{14}$, R.P. Haddock ${ }^{9}$, F.H. Heinsius ${ }^{1}$ b, M. Heinzelmann ${ }^{14}$, A. Herbstrith $^{8}$, \\ N.P. Hessey ${ }^{10}$, P. Hidas ${ }^{4}$, C. Holtzhaußen ${ }^{8}$, D. Jamnik ${ }^{10}$ c, H. Kalinowsky ${ }^{3}$, P. Kammel ${ }^{1}$, J. Kisiel ${ }^{6}$ d, E. Klempt ${ }^{3}$, \\ H. Koch ${ }^{2}$, M. Kunze ${ }^{2}$, U. Kurilla ${ }^{2}$, M. Lakata ${ }^{1}$, R. Landua ${ }^{6}$, H. Matthäy ${ }^{2}$, C.A. Meyer ${ }^{12}$, F. Meyer-Wildhagen ${ }^{10}$, \\ L. Montanet ${ }^{6}$, R. Ouared ${ }^{6}$, K. Peters ${ }^{2}$, B. Pick ${ }^{3}$, M. Ratajczak ${ }^{2}$, C. Regenfus ${ }^{14}$, W. Roethel ${ }^{10}$, S. Spanier ${ }^{14}$ e \\ H. Stöck ${ }^{2}$, U. Strohbusch ${ }^{7}$, M. Suffert ${ }^{13}$, J.S. Suh ${ }^{3}$, U. Thoma ${ }^{3}$, M. Tischhäuser ${ }^{8}$, I. Uman ${ }^{10}$, S. Wallis-Plachner ${ }^{10}$, \\ D. Walther ${ }^{10} \mathrm{~g}, \mathrm{U}$. Wiedner ${ }^{10 \mathrm{~h}}$, and K. Wittmack ${ }^{3}$ \\ 1 University of California, LBNL, Berkeley, CA 94720, USA \\ 2 Universität Bochum, D-44780 Bochum, Germany \\ 3 Universität Bonn, D-53115 Bonn, Germany \\ 4 Academy of Science, H-1525 Budapest, Hungary \\ 5 Rutherford Appleton Laboratory, Chilton, DidcotOX11 0QX, UK \\ ${ }^{6}$ CERN, CH-1211 Geneva 4, Switzerland \\ 7 Universität Hamburg, D-22761 Hamburg, Germany \\ 8 Universität Karlsruhe, D-76021 Karlsruhe, Germany \\ 9 University of California, Los Angeles, CA 90024, USA \\ 10 Universität München, D-80333 München, Germany \\ 11 LPNHE Paris VI, VII, F-75252 Paris, France \\ 12 Carnegie Mellon University, Pittsburgh, PA 15213, USA \\ 13 Centre de Recherches Nucléaires,F-67037 Strasbourg, France \\ 14 Universität Zürich, CH-8057 Zürich,Switzerland
}

January 4, 2001

\begin{abstract}
A simultaneous analysis of two different $5 \pi$ final states from $\bar{p} N$ annihilation at rest is used to study the decays of isoscalar $0^{++}$states into $4 \pi^{0}$. The data demand two scalar states, the $f_{0}(1370)$ with $m=1395 \pm 40 \mathrm{MeV} / \mathrm{c}^{2}, \Gamma=275 \pm 55 \mathrm{MeV} / \mathrm{c}^{2}$ and the $f_{0}(1500)$ with mass and width compatible with previous findings. Apart from the dominant scalar intensity, the $\pi(1300)$ plays an important role in the $5 \pi$ dynamics. We find a mass of $m=1375 \pm 40 \mathrm{MeV} / \mathrm{c}^{2}$ and a width of $\Gamma=268 \pm 50 \mathrm{MeV} / \mathrm{c}^{2}$ with a dominant decay into $\rho \pi$. The $(\pi \pi)_{s} \pi$ decay of the $\pi(1300)$ is less than $15 \%$ of its $\rho \pi$ decay.
\end{abstract}

\section{Introduction}

The spectrum of scalar mesons is of particular importance in meson spectroscopy since the ground-state glueball is predicted to have scalar quantum numbers and a mass of about $1.73 \mathrm{GeV} / \mathrm{c}^{2}[1]$. In particular the peculiar decay pattern of the $f_{0}(1500)$ into two pseudo-scalar mesons has led to the hypothesis that it may be the scalar glueball

${ }^{a}$ Now at University of Mainz, Mainz, Germany

b Now at University of Freiburg, Freiburg, Germany

c University of Ljubljana, Ljubljana, Slovenia

d University of Silesia, Katowice, Poland

e Now at SLAC, Stanford, USA

f This work comprises part of the $\mathrm{PhD}$ thesis of U.Thoma

$g$ Now at University of Bonn, Bonn, Germany

h Now at Uppsala University, Uppsala, Sweden mixed with normal scalar mesons [2-5]. Properties of all scalar states are obviously important for this interpretation.

The $f_{0}(1370)$ and $f_{0}(1500)$ and their decays into 2 pseudoscalar mesons have been studied in detail in $\bar{p} p$ annihilations at rest by the Crystal Barrel Collaboration [611 , but the $f_{0}(1370)$ has been clouded by a broad structure coupling to $\pi \pi$ and currently referred to as $f_{0}(400-$ 1200) [12]. While the data suggest the existence of two comparatively narrow states, $f_{0}(1370)$ and $f_{0}(1500)$, solutions with a broad structure instead of the $f_{0}(1370)$ are not excluded [7]. Also, in the analysis [13] of the reaction $\bar{p} p \rightarrow 5 \pi^{0}$, the $f_{0}(1370)$ was not unambiguously established. 
In this paper we present an analysis of the reaction

$$
\bar{p} d \rightarrow \pi^{-} 4 \pi^{0} p_{\text {spectator }}
$$

which has one distinctive advantage over the reaction studied before: in annihilations on neutrons there exists only one $4 \pi^{0}$ combination while in the $5 \pi^{0}$ final state there are five combinations which could make up an $f_{0}$ state. We also find that the solution obtained from reaction (1) gives a good description of the reaction $\bar{p} p \rightarrow 5 \pi^{0}$.

\section{The data analysis}

The data presented in this letter have been taken at the Low-Energy-Antiproton-Ring LEAR at CERN. Low energy antiprotons $(200 \mathrm{MeV} / \mathrm{c})$ are extracted and brought to rest in a liquid deuterium target. The annihilation products are then detected in the Crystal Barrel detector, which has been described in detail elsewhere [14]. Here, we mention only its main components. The target is surrounded by a pair of cylindrical multi-wire proportional chambers (PWC's) and a 23-layer cylindrical drift chamber (JDC). The momentum resolution for charged particle is $\delta p / p=6.5 \%$ at $1 \mathrm{GeV} / \mathrm{c}$. The JDC is in turn surrounded by a 1380 crystal CsI(Tl) barrel calorimeter. The crystals point towards the target center and the calorimeter covers polar angles between $12^{0}$ and $168^{\circ}$ degrees and $2 \pi$ in azimuth. The useful acceptance for shower detection is $95 \%$ of $4 \pi$. Typical photon energy resolutions are $\sigma_{E} / E \approx 2.5 \%$ at $1 \mathrm{GeV}$ and $\sigma_{\phi, \theta}=1.2^{0}$.

The data of the current analysis have been collected using a one-prong trigger. This trigger selects events with one track reaching the outermost layers of the JDC by requiring one hit in the PWC and one or two hits in the outer layers of the JDC. In these events the proton does not escape the target. The data are then reconstructed and those events satisfying the following criteria are retained:

- Exactly one negative charged track in the JDC.

- Exactly 8 photons with energies above $20 \mathrm{MeV}$.

- For each electromagnetic shower, the energy deposited in the central crystal should exceed $13 \mathrm{MeV}$. This removes spurious hits due to shower fluctuations.

- Events containing photons centered in the crystals adjacent to the beam pipe are rejected due to possible shower leakage.

Data surviving these cuts are then submitted to a 1constraint kinematic fit to the hypothesis $\bar{p} d \rightarrow \pi^{-} 8 \gamma$ plus a missing proton. In a second step, a series of higher constraint kinematic fits are performed in which the $\gamma \gamma$ pairs are constrained to be either $\pi^{0}$ or $\eta$. The best hypothesis is required to be $\pi^{-} 4 \pi^{0} p_{\text {spectator }}$ and the probability for this assignment must be larger than 10\%. Furthermore, all events with a spectator proton momentum larger than $100 \mathrm{MeV} / \mathrm{c}$ are rejected in order to select events in which the $\bar{p}$ annihilates on a quasi-free neutron. From the 5.2 million triggered one-prong events, 42738 survive these cuts. For these events, we form the three possible $3 \pi^{0}$ invariant masses and remove all events with an entry within
$30 \mathrm{MeV} / \mathrm{c}^{2}$ of the $\eta(548)$ mass. This reduces the number of events to 30016 . Monte Carlo studies show that background contributions from other channels are below $1 \%$.

The branching fraction for reaction (1) has been determined using minimum-bias data. The number of reconstructed $5 \pi$ events excluding $\pi^{-} \pi^{0} \eta\left[\rightarrow 3 \pi^{0}\right]$ is $N_{5 \pi}=457$ from a sample of $N_{m b}=1293538$ minimum bias events. The reconstruction efficiency is determined from the Crystal Barrel Monte Carlo program using GEANT. Identical cuts are applied as for real data and we find an efficiency of $\varepsilon_{m c}=(5.5 \pm 0.3) \%$ which includes the decay of the $\pi^{\circ}$ 's, $\left(\mathrm{BR}\left(\pi^{\circ} \rightarrow \gamma \gamma\right)=0.98797 \pm 0.00032\right)$. In addition, there is a correction for events which do not annihilate in the target, $\varepsilon_{1}=0.956 \pm 0.025$. From these numbers we derive our final branching ratio excluding contributions from $\bar{p} d \rightarrow \pi^{-} \pi^{0} \eta,\left(\eta \rightarrow 3 \pi^{0}\right)$.

$$
\mathrm{BR}\left(\bar{p} d \rightarrow \pi^{-} 4 \pi^{0} p\right)=\frac{N_{5 \pi}}{\varepsilon_{m c} \cdot \varepsilon_{1} \cdot N_{m b}}=(0.67 \pm 0.10) \% .
$$

In Fig. 1 we show the $4 \pi, 3 \pi$ and $2 \pi$ invariant mass combinations together with the phase-space distributions. The most striking feature is a relatively narrow peak in the $4 \pi^{0}$ invariant mass spectrum (a) centered at $m=1.48 \mathrm{GeV} / \mathrm{c}^{2}$ which is not observed in the $3 \pi^{0} \pi^{-}$invariant mass distribution (b). In the partial wave analysis described below the peak in (a) is found to be dominantly due to $f_{0}(1370)$ and $f_{0}(1500)$ production and subsequent decay into $4 \pi^{0}$. In the $\pi^{-} \pi^{0}$ invariant mass distribution, (f), evidence is seen for the $\rho^{-}(770)$. It is produced as a secondary particle from production of e.g. $\pi^{-}(1300) \rightarrow \rho^{-} \pi^{0}$. Indeed, the $\pi^{-} \pi^{0} \pi^{0}$ invariant mass plot (d) shows a broad high-mass shoulder at $\sim 1300 \mathrm{MeV} / \mathrm{c}^{2}$ which is not visible in the $3 \pi^{0}$ invariant mass plot $(\mathbf{c})$.

\section{The partial wave analysis}

\subsection{The amplitudes}

The data are analyzed in terms of the isobar model [16]. The initial $\bar{p} n$ system is assumed to decay to the final state through a series of quasi two-body decays via intermediate resonances $A, B$ and $C$. We consider the following decay chains:

$$
\begin{aligned}
& \bar{p} d \rightarrow A \pi \rightarrow\{B C\} \pi \rightarrow\{(\pi \pi)(\pi \pi)\} \pi, \\
& \bar{p} d \rightarrow A \pi \rightarrow\{B \pi\} \pi \rightarrow\{[(\pi \pi) \pi] \pi)\} \pi \text { and } \\
& \bar{p} d \rightarrow A C \rightarrow\{B \pi\} C \rightarrow\{(\pi \pi) \pi\}(\pi \pi) .
\end{aligned}
$$

The amplitude for such a reaction can be written as:

$$
\mathcal{A}_{\text {initial state } i}=\sum_{k=1}^{n \text { amplitudes }} \beta_{k} \mathcal{A}_{k}
$$

were $\beta_{k}$ is a complex value parameterizing the production of each amplitude $\mathcal{A}_{k}$. $\mathcal{A}_{k}$ describes the production and decay of the resonances involved. In general, the $\bar{p} p$ initial states contribute incoherently to the final state. In this analysis, we restrict ourselves to annihilation from the 

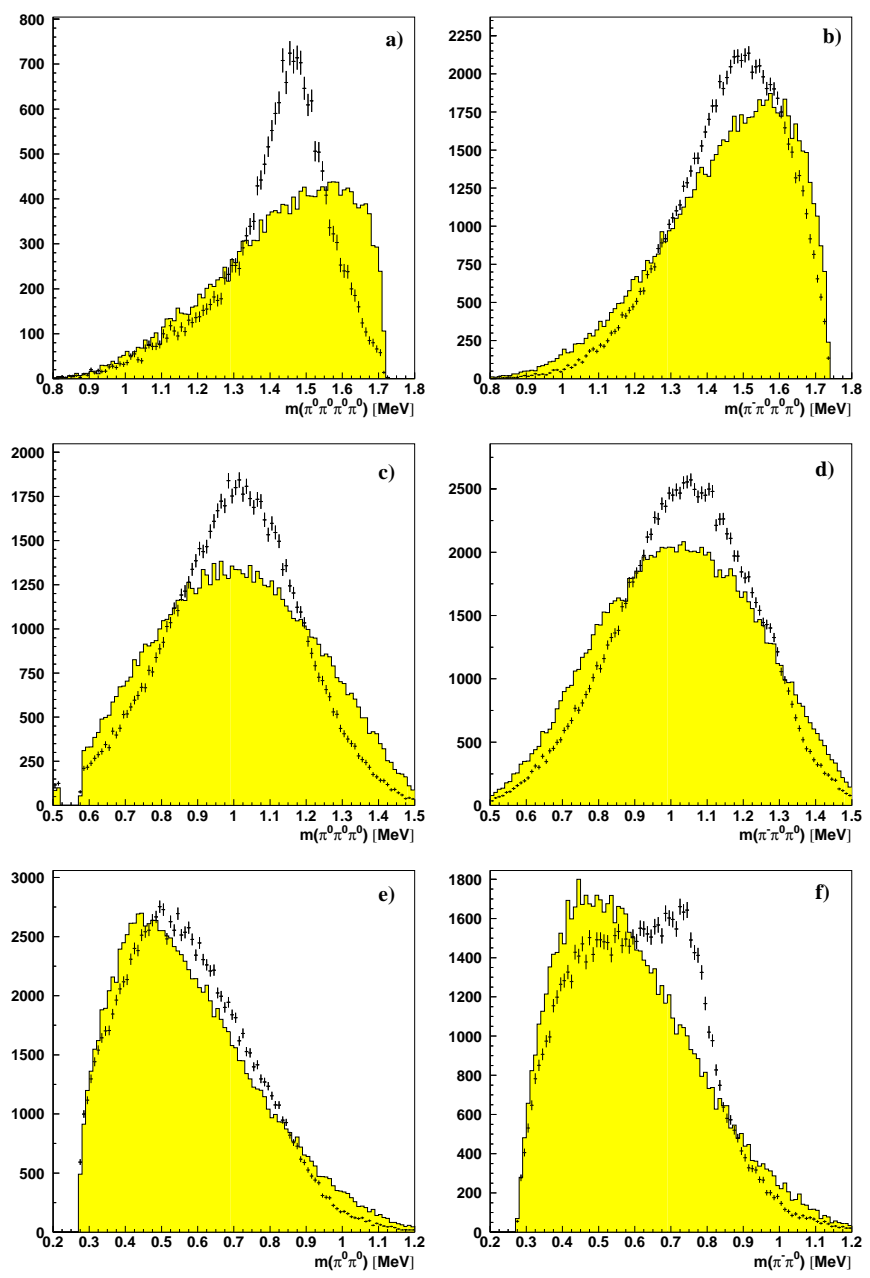

Fig. 1. The possible invariant mass combinations in the $\pi^{-} 4 \pi^{0}$ data set. (a) $4 \pi^{0}$ system, (one combination per event). (b) $\pi^{-} 3 \pi^{0}$ system, (four combinations per event). (c) $3 \pi^{0}$ system, (four combinations per event). (d) $\pi^{-} 2 \pi^{0}$ system, (six combinations per event). (e) $2 \pi^{0}$ system, (six combinations per event). (f) $\pi^{-} \pi^{0}$ system, (four combinations per event). The data are shown with statistical error bars, while the shaded curves are phase space distributions for $\bar{p} n \rightarrow \pi^{-} 4 \pi^{0}$.

${ }^{1} S_{0}$ initial state, as ${ }^{3} S_{1}$ is not allowed by isospin and $G$ parity conservation. This approximation is justified for $\bar{p} p$ annihilation [19] but for annihilation in liquid deuterium we should expect contributions from the ${ }^{3} P_{0},{ }^{3} P_{1}$ and ${ }^{3} P_{2}$ states. The inclusion of $P$-states is, however, not possible because of the large number of additional waves. The consistency of the results from $\bar{p} p$ (with a much smaller contribution from initial $P$-states) and $\bar{p} n$ annihilations may justify this approximation. We note that scalar and pseudoscalar mesons do not carry any information about the initial $\bar{p} N$-states and the vector mesons contribute only at the level of less than $15 \%$; their largest fraction stems from the ${ }^{1} S_{0}$ initial state [20]. Tests have been performed introducing amplitudes from $P$-states to check their influence on the other amplitudes which was found to be negligible. We conclude that neglecting $P$-state annihilation does not lead to incorrect conclusions for masses and widths of intermediate resonances.

The partial wave analysis attempts to explain the data with the smallest possible number of amplitudes. The amplitudes introduced for fitting the two data sets are summarized in Table 1. Throughout this paper we will treat the isospin zero $J^{P C}=0^{++} \pi \pi$ scattering amplitude, $(\pi \pi)_{S}$ as one isobar. In Table 1 the short-hand notation $\sigma$ is used for this amplitude. We do not interpret the $\sigma$ as genuine resonance. We use the energy dependent amplitude describing the $\pi \pi$ interaction from [10].

The amplitudes $\mathcal{A}_{k}$ have the general form:

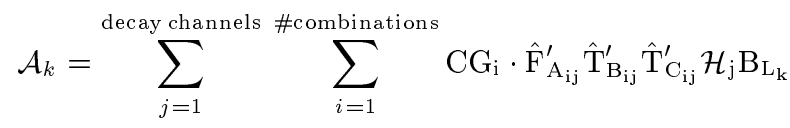

The production and decay of the first resonance is described by the production vector $\hat{F}=\beta \cdot \hat{F}^{\prime} ; \hat{T}^{\prime}$ describes the decays of the daughter products. $\mathcal{H}_{j}$ denotes the angular distribution which is calculated using the helicity formalism [18] and $B_{L_{k}}$ describes the centrifugal barrier of the production of the first resonance. The Clebsch-Gordan coefficients $C G_{i}$ have to be taken into account for every possible combination of the final state pions. Assuming two open decay channels for the produced resonance, $\hat{F}^{\prime}$ has the form:

$$
\begin{aligned}
& \hat{F}^{\prime}=\frac{m_{0} \cdot \sqrt{\Gamma_{0}}}{m_{0}{ }^{2}-m^{2}-i m_{0}\left[\frac{\rho_{1}(m)}{\rho_{1}^{0}} \Gamma_{1} B_{L 1}^{2}+\frac{\rho_{2}(m)}{\rho_{2}^{0}} \Gamma_{2} B_{L 2}^{2}\right]} \times \\
& \times\left(\begin{array}{l}
\sqrt{\frac{\Gamma_{1}}{\rho_{1}^{0}}} B_{L 1} \\
\sqrt{\frac{\Gamma_{2}}{\rho_{2}^{0}}} B_{L 2}
\end{array}\right)
\end{aligned}
$$

$\hat{T}^{\prime}$ looks very similar, namely:

$$
\hat{T}_{1}^{\prime}=\frac{m_{0} \cdot \sqrt{\Gamma_{0} \cdot \Gamma_{1} / \rho_{1}^{0}} \cdot B_{L 1}}{m_{0}^{2}-m^{2}-i m_{0}\left[\frac{\rho_{1}(m)}{\rho_{1}^{0}} \Gamma_{1} B_{L 1}^{2}+\frac{\rho_{2}(m)}{\rho_{2}^{0}} \Gamma_{2} B_{L 2}^{2}\right]},
$$

where $m_{0}$ and $\Gamma_{0}$ are the nominal mass and width of the resonance, and the $\rho$ 's are the phase space factors. For more than two decay channels $(5),(6)$ can be extended using additional terms $\frac{\rho_{i}(m)}{\rho_{i}^{0}} \cdot \Gamma_{i} \cdot B_{L i}^{2}$ in the denominator and a corresponding term in the numerator, where $B_{L i}$ are the centrifugal barrier factors for the decay of the resonances in the respective channels. If $\hat{T}^{\prime}$ is replaced by a $\hat{T}$-matrix ( numerator in (6) changed to $m_{0} \cdot\left(\Gamma_{1} / \rho_{1}^{0}\right) \cdot B_{L 1}^{2}$ ) assuming that the second-step resonances are produced by scattering, the influence on the resonance masses and widths is found to be negligible. The effects on the branching ratios are within the errors given. Contributions of amplitudes including a $B_{L}^{2}$ not equal zero in the T-matrices are slightly decreased using this parametrisation.

For the scalar resonances we use two partially overlapping Breit-Wigner amplitudes. It is well known that overlapping Breit-Wigner amplitudes may lead to a violation 


\begin{tabular}{clccc}
\hline \multicolumn{1}{c}{ Amplitude } & \multicolumn{1}{c}{ Decay } & $5 \pi^{0}$ & $\pi^{-} 4 \pi^{0}$ & Possible Resonances \\
\hline $\bar{p} N\left({ }^{1} S_{0}\right) \rightarrow f_{0} \pi ;$ & $f_{0} \rightarrow \sigma \sigma$ & $\star$ & $\star$ & \\
$\bar{p} N\left({ }^{1} S_{0}\right) \rightarrow f_{0} \pi ;$ & $f_{0} \rightarrow \pi^{*}[\rightarrow \sigma \pi] \pi$ & $\star$ & $\star$ & $f_{0}(1370), f_{0}(1500), f_{0}(1700)$ \\
\hline $\bar{p} N\left({ }^{1} S_{0}\right) \rightarrow \rho^{\prime} \pi ;$ & $\rho^{\prime} \rightarrow \rho \sigma ;$ & & $\star$ & \\
$\bar{p} N\left({ }^{1} S_{0}\right) \rightarrow \rho^{\prime} \pi ;$ & $\rho^{\prime} \rightarrow a_{1}[\rightarrow \rho \pi] \pi$ & & $\star$ & \\
$\bar{p} N\left({ }^{1} S_{0}\right) \rightarrow \rho^{\prime} \pi ;$ & $\rho^{\prime} \rightarrow \pi^{*}[\rightarrow \sigma \pi] \pi$ & & $\star$ & \\
$\bar{p} N\left({ }^{1} S_{0}\right) \rightarrow \rho^{\prime} \pi ;$ & $\rho^{\prime} \rightarrow \pi^{*}[\rightarrow \rho \pi] \pi$ & & $\star$ & $\rho(1450), \rho(1700)$ \\
\hline $\bar{p} N\left({ }^{1} S_{0}\right) \rightarrow \pi^{*} \sigma ;$ & $\pi^{*} \rightarrow \sigma \pi$ & $\star$ & $\star$ & \\
$\bar{p} N\left({ }^{1} S_{0}\right) \rightarrow \pi^{*} \sigma ;$ & $\pi^{*} \rightarrow \rho \pi$ & & $\star$ & \\
$\bar{p} N\left({ }^{1} S_{0}\right) \rightarrow \pi^{*} \rho ;$ & $\pi^{*} \rightarrow \sigma \pi$ & & $\star$ & $\pi(1300)$ \\
\hline $\bar{p} N\left({ }^{1} S_{0}\right) \rightarrow a_{1} \sigma ;$ & $a_{1} \rightarrow \rho \pi$ & & $\star$ & $a_{1}(1260)$ \\
\hline $\bar{p} N\left({ }^{1} S_{0}\right) \rightarrow f_{2} \pi ;$ & $f_{2} \rightarrow \sigma \sigma$ & $\star$ & $\star$ & $f_{2}(1270), f_{2}(1565)$ \\
\hline
\end{tabular}

Table 1. The amplitudes used to fit the $\pi^{-} 4 \pi^{0}$ and $5 \pi^{0}$ data sets. The $\star^{\prime}$ s in the $5 \pi^{0}$ and $\pi^{-} 4 \pi^{0}$ columns indicate which amplitudes are possible in each final state. The $\sigma$ represents the $(\pi \pi)_{s}$ wave and the $\pi^{*}$ represents the $\pi(1300)$.

of unitarity. In references [6-11] a $P$-vector ansatz based on the $K$-matrix approach [17] was therefore used. For a decay like $f_{0}(1500) \rightarrow \sigma \sigma$, there is no $K$-matrix description of the amplitude. We use a Breit-Wigner parametrisation allowing for different partial widths, see eqns. (5), (6). The two tensor states $f_{2}(1270)$ and $f_{2}(1565)$ are also described in this way, with a Blatt-Weisskopf barrier factor squared, $B^{2}\left(q, q_{0}\right)[17]$.

In addition to the (dominant) scalar intensity we find that $\pi(1300)(\pi \pi)_{s}$ plays an important role in describing the data. We have included the $\pi(1300)$ with decays into both $\rho \pi$ and $(\pi \pi)_{s} \pi$ using a modified Breit-Wigner for the two decays into $\rho \pi$ and $(\pi \pi)_{s} \pi$ decays. In this formulation, we rewrite the partial width of a decay in terms of a normalized scale parameter $\gamma_{x x}^{2}=\Gamma_{x x} /\left(\Gamma_{0} \rho_{x x}^{0}\right)$, and the resonance parameterisation is given by:

$$
\begin{aligned}
& \mathrm{BW}_{\rho \pi}= \\
& \frac{\gamma_{\rho \pi} m_{0} \Gamma_{0} B^{1}\left(q, q_{0}\right)}{m_{0}^{2}-m^{2}-i m_{0} \Gamma_{0}\left[\rho_{1}(m) \gamma_{(\pi \pi)_{s} \pi}^{2}+\left(B^{1}\left(q, q_{0}\right)\right)^{2} \rho_{2}(m) \gamma_{\rho \pi}^{2}\right]} \\
& \mathrm{BW}_{(\pi \pi)_{s} \pi}= \\
& \frac{\gamma_{(\pi \pi)_{s} \pi} m_{0} \Gamma_{0}}{m_{0}^{2}-m^{2}-i m_{0} \Gamma_{0}\left[\rho_{1}(m) \gamma_{(\pi \pi)_{s} \pi}^{2}+\left(B^{1}\left(q, q_{0}\right)\right)^{2} \rho_{2}(m) \gamma_{\rho \pi}^{2}\right]}
\end{aligned}
$$

The nominal mass and width of the $\pi(1300)$ are $m_{0}$ and $\Gamma_{0}$, respectively, $m$ is the actual mass, $q$ is the decay momentum of the lone $\pi$ recoiling against the $\rho$ or the $(\pi \pi)_{s}-$ wave in the $\pi(1300)$ rest-frame at a 3 -pion-mass $m, q_{0}$ is computed at the mass $m_{0}$. The phase space factor is $\rho(m)=q / 2 m$, and $B^{1}\left(q, q_{0}\right)$ is the ratio of barrier factor evaluated at $q$ over the barrier factor evaluated at $q_{0}$. Corresponding amplitudes are defined to describe production and decay of $\rho$-states (denoted as $\rho^{\prime}$ in Table 1 ) decaying into four pions. The $a_{1}(1230)$ is described by a onechannel Breit-Wigner amplitude.

An unbinned maximum likelihood fit is used, where the quantity $-2 \log \mathcal{L}$ is minimized using the MINUIT program. This procedure fits the full 8 -dimensional phase-space of the $5 \pi$ final state. A sample of 22000 Monte Carlo events which have survived exactly the same cuts as the real data are used for normalization.

\subsection{Fits to the $\pi^{-} 4 \pi^{0}$ data set}

The minimal hypotheses assumes one scalar resonance decaying into $(\pi \pi)_{s}(\pi \pi)_{s}$ and a $\rho^{-}(1450)$ decaying into $\rho(\pi \pi)_{s}$. This fit yields a scalar mass and width of $m \approx 1400 \mathrm{MeV} / \mathrm{c}^{2}$ and $\Gamma \approx 250 \mathrm{MeV} / \mathrm{c}^{2}$ which is consistent with earlier publications on $\bar{p} N$ annihilation into five pions [23-25]. However, the data are not well described, particularly in the peak region of the $4 \pi^{0}$ invariant mass. The introduction of an additional scalar resonance, $f_{0}(1500)$, leads to a large improvement of 300 in $2 \ln (\mathcal{L})$ and a superior description of the $4 \pi^{0}$ peak region.

Next we introduce the amplitude for $\bar{p} n \rightarrow \pi(1300)(\pi \pi)_{s}$, fitting the $\gamma^{2}$ terms in eqns (7) and (8) which describe the decay rate into the two final states. This leads to an improvement of 1900 in $2 \ln \mathcal{L}$ which is a highly significant change. From this fit, we find that $\gamma_{(\pi \pi) \pi}^{2}=0.01 \pm 0.01$ and $\gamma_{\rho \pi}^{2}=0.99 \pm 0.01$. This result indicates that the dominant decay of the $\pi(1300)$ is $\rho \pi$. Based on a systematic study of these quantities, we find an upper limit on the decay into $(\pi \pi)_{s} \pi$ as:

$$
\frac{\mathrm{BR}\left[\pi(1300) \rightarrow(\pi \pi)_{s} \pi\right]}{\operatorname{BR}[\pi(1300) \rightarrow \rho \pi]}<0.15
$$

This can be seen in Fig. 2,3 where we plot $2 \ln \mathcal{L}$ as a function of the $\pi(1300)$ mass for both decay modes. The $\rho \pi$ decay shows a pronounced peak near a mass of $1400 \mathrm{MeV} / \mathrm{c}^{2}$ and a width near $270 \mathrm{MeV} / \mathrm{c}^{2}$. The same plot for the $(\pi \pi)_{s} \pi$ decay shows no evidence for the $\pi(1300)$. Based on this, we have only included the $\pi(1300) \rightarrow \rho \pi$ decay in the final analysis. All amplitudes in Table 1 involving $\pi(1300) \rightarrow(\pi \pi)_{s} \pi$ are neglected.

Now we consider the $\rho(1450)$ and $\rho(1700)$. Both of these states have been observed in $\bar{p} p$-annihilation via 


\begin{tabular}{lll}
\hline \multicolumn{1}{c}{ Amplitude } & \multicolumn{1}{c}{ Parameters } & fraction \\
\hline$f_{0}^{(1)} \pi, f_{0} \rightarrow(\pi \pi)_{S}(\pi \pi)_{S}$ & $m=1395 \pm 40 \mathrm{MeV} / \mathrm{c}^{2}$, & \\
& $\Gamma=275 \pm 55 \mathrm{MeV} / \mathrm{c}^{2}$ & $68.9 \pm 4 \%$ \\
$f_{0}^{(2)} \pi, f_{0} \rightarrow(\pi \pi)_{S}(\pi \pi)_{S}$ & $m=1490 \pm 30 \mathrm{MeV} / \mathrm{c}^{2}$, & \\
& $\Gamma=140 \pm 40 \mathrm{MeV} / \mathrm{c}^{2}$ & $6.7 \pm 0.7 \%$ \\
$f_{0}^{(3)} \pi, f_{0} \rightarrow(\pi \pi)_{S}(\pi \pi)_{S}$ & $m=1770 \mathrm{MeV} / \mathrm{c}^{2 \dagger}, \Gamma=170 \mathrm{MeV} / \mathrm{c}^{2 \dagger}$ & $0.5-3 \%$ \\
$\pi(1300)(\pi \pi)_{S}, \pi(1300) \rightarrow \rho \pi$ & $m=1375 \pm 40 \mathrm{MeV} / \mathrm{c}^{2}$, & \\
& $\Gamma=268 \pm 50 \mathrm{MeV} / \mathrm{c}^{2}$ & $5.4 \pm 0.4 \%$ \\
$\rho(1450) \pi ; \rho(1700) \pi$ & $m=1435 \mathrm{MeV} / \mathrm{c}^{2 \dagger}, \Gamma=325 \mathrm{MeV} / \mathrm{c}^{2 \dagger}$ & $3.6 \pm 0.5 \%$ \\
& $m=1700 \mathrm{MeV} / \mathrm{c}^{2 \dagger}, \Gamma=235 \mathrm{MeV} / \mathrm{c}^{2 \dagger}$ & $10.1 \pm 1.2 \%$
\end{tabular}

Table 2. The fit results for the $\pi^{-} 4 \pi^{0}$ data. The masses and widths marked with a ' $\dagger$ ' have been held fixed at the given value.

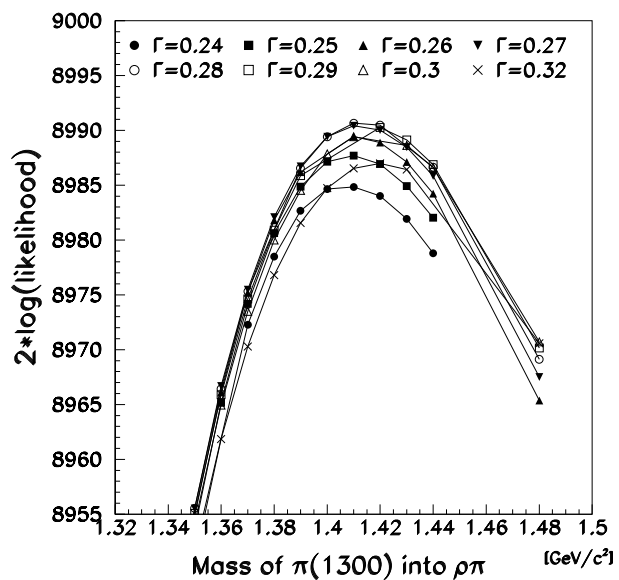

Fig. 2. Scan of the $\pi(1300)$ mass and width, only $\pi(1300) \rightarrow$ $\rho \pi$ decay allowed. All amplitudes given in Table 2 have been introduced.

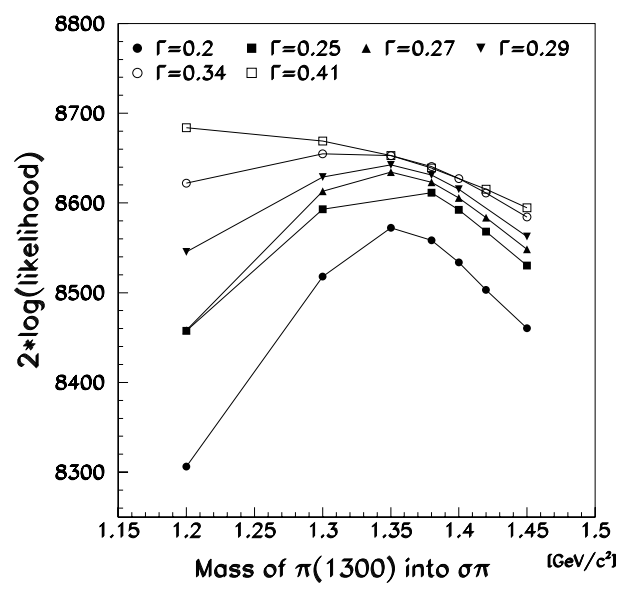

Fig. 3. Scan of the $\pi(1300)$ mass and width, only $\pi(1300) \rightarrow$ $\sigma \pi$ decay allowed. All amplitudes given in Table 2 have been introduced. their $2 \pi$ decays in the $\pi^{-} \pi^{0} \pi^{0}$ final state [15]. In this analysis we allowed for the decay modes given in Table 1 . Both $\rho$ states contribute at the few percent level but we are not sensitive to the mass and width of either the $\rho(1450)$, $\rho(1700)$ or the $a_{1}(1230)$. The fit finds broad and shallow maxima in $2 \ln \mathcal{L}$ consistent with the $\rho$ parameters from our $2 \pi$ studies and with the world average for the $\rho$ and $a_{1}$ parameters [12] with no sensitivity to the precise values. Hence we fix the parameters to the mean values of [12] and [15]. The effect of these additional amplitudes increases $2 \ln \mathcal{L}$ by about 600 . A detailed discussion of the different decay modes of these states will be given elsewhere [26], including the information from a third $5 \pi$ final state which allows for additional decay modes.

After introduction of all the amplitudes given in Table 1, we return to the discussion of the scalar states. In particular, we have tried to describe the data using only one $f_{0}$ state decaying into $4 \pi^{0}$. Figure 4 shows the data for the $4 \pi^{0}$ invariant mass. Superimposed is the best fit with one $f_{0}$ and that with two $f_{0}$ states. Both the peak region and the high mass edge of the peak are poorly described with only one scalar state. This is significantly improved with the introduction of a second scalar state. The fit to the mass and width of these two states optimize at $m=1395 \pm 40 \mathrm{MeV} / \mathrm{c}^{2}$ and $\Gamma=275 \pm 55 \mathrm{MeV} / \mathrm{c}^{2}$ for the
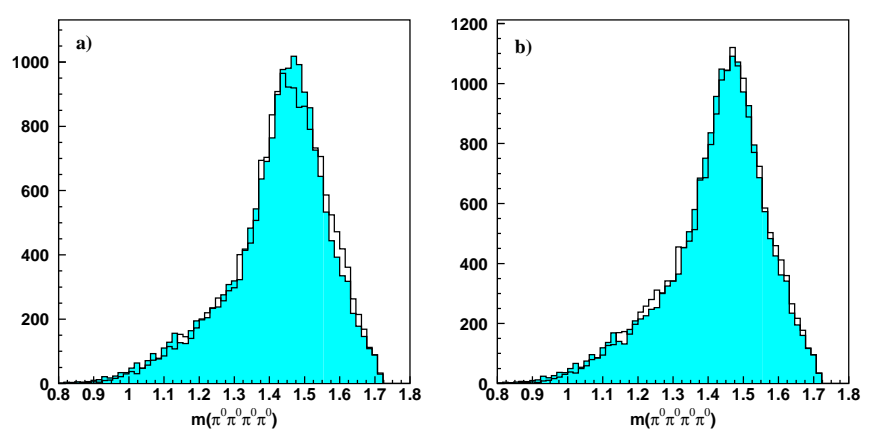

Fig. 4. The $4 \pi^{0}$ invariant mass from the $\pi^{-} 4 \pi^{0}$ final state. The shaded distribution shows the data, the line the best fit with a) only one $f_{0}, \mathrm{~b}$ ) two $f_{0}$-states allowed. 
lighter state and to $m=1490 \pm 30 \mathrm{MeV} / \mathrm{c}^{2}$ and $\Gamma=140 \pm 40 \mathrm{MeV} / \mathrm{c}^{2}$ for the heavier state. The maximum in $2 \ln \mathcal{L}$ are shown in Fig. 5,6 for the two scalar states.

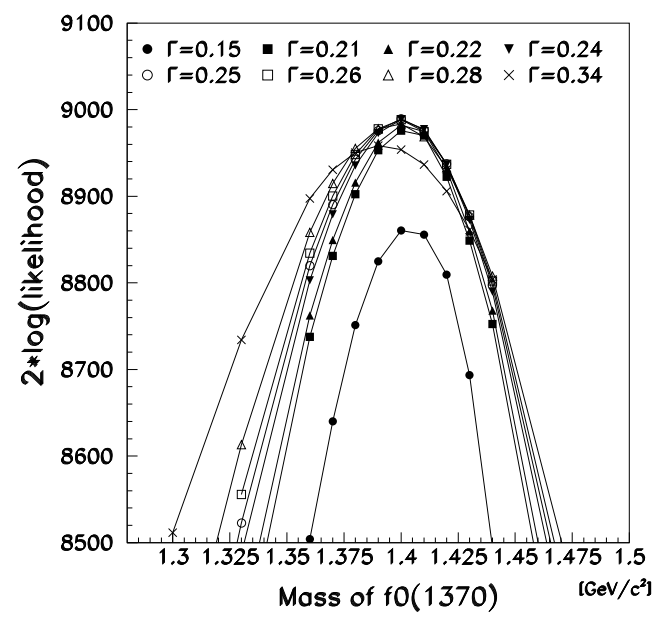

Fig. 5. Scan of the $f_{0}(1370)$ mass and width. All other amplitudes given in Table 2 have been introduced with fixed masses and widths.

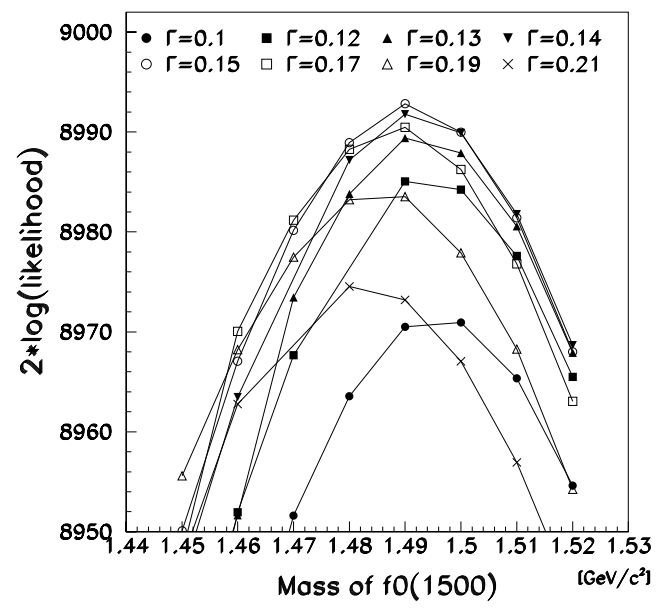

Fig. 6. Scan of the $f_{0}(1500)$ mass and width. All other amplitudes given in Table 2 have been introduced with fixed masses and widths.

The $f_{0}(1500)$ width is consistent within statistical errors with the PDG value of $112 \mathrm{MeV} / \mathrm{c}^{2}$ [12]. However, these fits tend to prefer a larger width, therefore we use a width of $130 \mathrm{MeV} / \mathrm{c}^{2}$ throughout this analysis. We have also introduced an $f_{0}(1770)$ state with mass and width taken from [27], $m=1770 \mathrm{MeV} / \mathrm{c}^{2}$ and $\Gamma=170 \mathrm{MeV} / \mathrm{c}^{2}$. The state leads to a very small improvement in $2 \ln \mathcal{L}$, and a slightly better description of the data in the high mass $4 \pi^{0}$ region, but the data are not sensitive to its mass and width. The introduction of this state with free parameters tends to destabilize the remainder of the fit and we are unable to establish if it is present or not. If the $f_{0}(1370)$ given above is replaced by direct $\sigma \sigma \pi$ production or a very broad resonance decaying into $\sigma \sigma$, like the $f_{0}(400-1200)$, $2 \ln (\mathcal{L})$ gets worse by more than 400 . Further tests have been made introducing a direct $\rho \sigma \pi$ - or $\sigma \sigma \pi$-amplitude in addition to the $f_{0}(1370)$ and the $f_{0}(1500)$. In the first case the change in $2 \ln (\mathcal{L})$ is insignificant and the influence of the additional amplitude on masses and widths is found to be negligible, the contributions of the different states are within the errors given. Introducing a direct $\sigma \sigma \pi$-amplitude leads to an improvement of 120 in $2 \ln (\mathcal{L})$ but the fits have a bad convergence behaviour. If the parameters of the $f_{0}$-states are left free, the mass and width of the $f_{0}(1500)$ found are no longer in agreement with our previous findings [6-11]. Therefore we consider this solution as unphysical.

Table 2 summarises the results of the best fit to the $\pi^{-} 4 \pi^{0}$ dataset. All fractions are calculated excluding the interference with the recoil meson, as explained in $[13,26]$; they do not necessarily add up to $100 \%$. The contribution of the $a_{1}(1230)(\pi \pi)_{s}$-amplitude is found to be negligible. Also the contribution of both $f_{2}(1270) \pi$ and $f_{2}(1565) \pi$ with the $f_{2}$ 's decaying into

$(\pi \pi)_{s}(\pi \pi)_{s}$ are found to be less than $0.5 \%$. This is consistent with the known production and decay rates of the $f_{2}(1270)$.

Several projections of the data are compared with the best fit in Fig. 7. The quality of the fit is in general very good, most of the visible deviations from the data are certainly within errors (the errors in the fit are not shown, but are of similar size to that of the data). The most noticeable deviation is in the $\pi^{-} \pi^{0}$ invariant mass where the fit underestimates the $\rho^{-}$peak. We have not found a consistent interpretation of the stronger $\rho^{-}$in the data. A better description can be found by incoherently adding any small amplitude including a $\rho^{-}$(e.g. an amplitude where the annihilation occurs from an initial $P$-state), but without a full treatment of the $P$-states, this seems to be rather ad hoc. In any case, the results of this paper are not affected by such an inclusion.

In addition to the amplitudes mentioned in Table 1, a possible $a_{1}(1230) \pi$ decay mode of the scalar states has been tested, using an $a_{1}(1230)$ fixed to PDG values for mass and width. The fit accepts a small contribution of the $f_{0}(1370)$ decaying into $a_{1}(1230)[\rightarrow \sigma \pi] \pi$ (less then $4 \%$ of its $\sigma \sigma$-decay) but the parameters of the $a_{1}(1230)$ itself are not stable in the fit. The introduction of an $f_{0}(1500) \rightarrow a_{1}(1230)[\rightarrow \sigma \pi] \pi$ amplitude does not lead to an improvement. When this decay is included in the fit to the $5 \pi^{0}$ data as discussed in the next section, it improves the description of the data slightly, but the results are inconsistent with the $\pi^{-} 4 \pi^{0}$ results. We conclude that there is no evidence for this decay mode. 

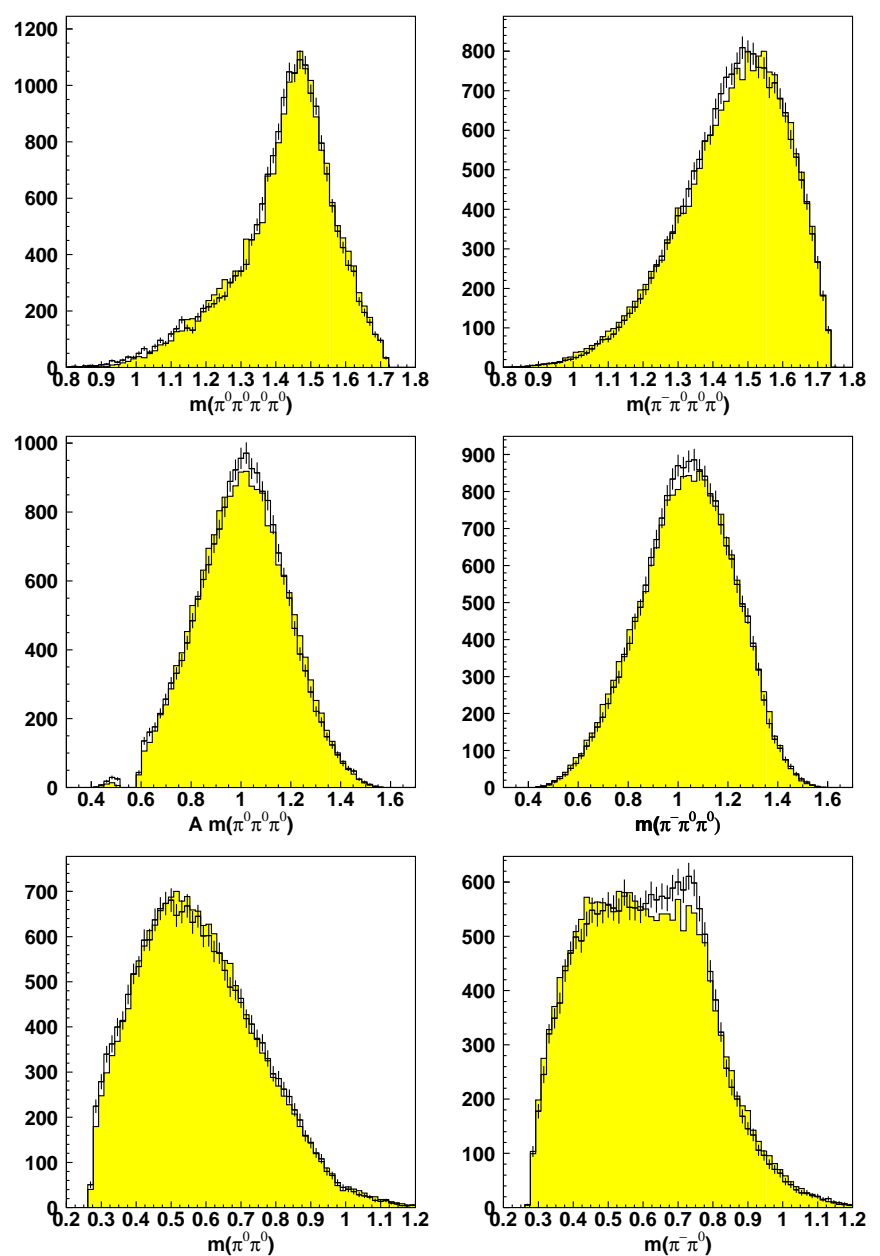

Fig. 7. A comparison between the data, (points with error bars), and the fit, (shaded region), to the $\pi^{-} 4 \pi^{0}$ data set. The effect of the $\eta$-anti-cut in the $3 \pi^{0}$-invariant mass is clearly visible.

\subsection{The reaction $\bar{p} p \rightarrow 5 \pi^{0}$}

Table 1 shows that all amplitudes contributing to the $\bar{p} p \rightarrow 5 \pi^{0}$ data [13] contribute also to $\bar{p} n \rightarrow \pi^{-} 4 \pi^{0}$. We have therefore reanalyzed the former data by introducing the masses and the total and partial decay widths of the contributing resonances found in this analysis. We have found that it is not only possible to fix the masses and widths but also the absolute ratios of the relative production strengths $\beta$ of the scalar states.

If one compares reactions (10) and (11)

$$
\begin{aligned}
\bar{p} n\left({ }^{1} S_{0}\right) & \rightarrow \pi^{-} f_{0}, f_{0} \rightarrow 4 \pi^{0} \\
\bar{p} p\left({ }^{1} S_{0}\right) & \rightarrow \pi^{0} f_{0}, f_{0} \rightarrow 4 \pi^{0}
\end{aligned}
$$

one would expect no difference between the relative production strengths $|\beta|$ of the different resonances if the same decay modes appear in both final states. This is not necessarily true for the phase of the $\beta$ 's since interferences or rescattering effects can be different in the two reactions.
If one leaves the relative production strengths free in the fit, they change by less than $20 \%$ from the values found from the $\pi^{-} 4 \pi^{0}$ data, depending on the exact values chosen for mass and width of the contributing $f_{0}$-states. The description of the data reached by fixing all masses, widths, partial widths and in addition the relative production strength to the values from fitting $\pi^{-} 4 \pi^{0}$ data set is shown in Fig. 8. The description is excellent. The dominant contributions in the $5 \pi^{0}$ data are two scalar states decaying into $(\pi \pi)_{s}(\pi \pi)_{s}$, the $f_{0}(1370)(37-42 \%)$ and the $f_{0}(1500)$ $(3-6 \%)$. The contribution of tensor states decaying into $(\pi \pi)_{s}(\pi \pi)_{s}$ is again below $0.5 \%$. The introduction of a third $f_{0}(1770)$ leads to a small improvement in the high mass region of the $4 \pi^{0}$ spectrum with a contribution of about $1 \%$. The branching fractions are computed ignoring interferences with the recoiling meson and do not add up to $100 \%$.


Fig. 8. Quality of the fit to the $5 \pi^{0}$ data. Masses, widths and the relative production strength of the particles have been fixed to the results found in fitting the $\pi^{-} 4 \pi^{0}$ data set. The data are shown as a line with error bars while the fit is the shaded area. 
For the total branching ratios of the two scalar states we find:

$$
\begin{gathered}
\mathrm{BR}\left(\bar{p} p \rightarrow f_{0}(1370) \pi^{0} \rightarrow(\pi \pi)_{S}(\pi \pi)_{S} \pi^{0} \rightarrow 5 \pi^{0}\right)= \\
(28.4 \pm 6.0) \cdot 10^{-4} \\
\operatorname{BR}\left(\bar{p} p \rightarrow f_{0}(1500) \pi^{0} \rightarrow(\pi \pi)_{S}(\pi \pi)_{S} \pi^{0} \rightarrow 5 \pi^{0}\right)= \\
(2.8 \pm 1.5) \cdot 10^{-4}
\end{gathered}
$$

These can be combined with an earlier analysis on the $3 \pi^{0}$ final states to obtain information on the relative branching ratios.

Using the result from reference [28] of

$$
\begin{array}{r}
\mathrm{BR}\left[\bar{p} p \rightarrow f_{0}(1370) \pi^{0}, f_{0}(1370) \rightarrow \pi^{0} \pi^{0}\right]= \\
(6.4 \pm 2.4) \cdot 10^{-4},
\end{array}
$$

we find the relative rate:

$$
\frac{f_{0}(1370) \rightarrow(\pi \pi)_{s}(\pi \pi)_{s} \rightarrow 4 \pi^{0}}{f_{0}(1370) \rightarrow 2 \pi^{0}}=4.4 \pm 1.9
$$

The $(\pi \pi)_{s}(\pi \pi)_{s}$ decay of the $f_{0}(1370)$ is significantly larger than the $\pi^{0} \pi^{0}$ mode. The dominance of the $4 \pi$ decay modes compared to its decay into two pseudoscalar states becomes even more obvious including other modes like $\rho \rho$, $\pi^{*}(1300) \pi$ and $a_{1} \pi$ [26]. Using the results on the $2 \pi$ decays of the $f_{0}(1500)$ from reference [28]

$$
\begin{aligned}
& \mathrm{BR}\left[\bar{p} p \rightarrow f_{0}(1500) \pi^{0},\right.\left.f_{0}(1500) \rightarrow \pi^{0} \pi^{0}\right]= \\
&(8.2 \pm 0.9) \times 10^{-4}
\end{aligned}
$$

we find the following relative rates for the $f_{0}(1500)$ :

$$
\frac{f_{0}(1500) \rightarrow(\pi \pi)_{s}(\pi \pi)_{s} \rightarrow 4 \pi^{0}}{f_{0}(1500) \rightarrow 2 \pi^{0}}=0.34 \pm 0.19
$$

In [26] additional $4 \pi$-decay modes of scalar states e.g. $\rho \rho, \pi^{*}[\rightarrow \rho \pi] \pi$ and $a_{1}[\rightarrow \rho \pi] \pi$ are investigated. The resulting branching ratios are summarized in Table 3 together with the branching ratios for the decays into two pseudoscalar states. The $4 \pi$ rates take into account the isospin Clebsch-Gordan coefficients. The role and treatment of interference effects are discussed in [26].

The $4 \pi$-decays of the $f_{0}(1370)$ are the dominant decay modes; in particular the two decays into $\sigma \sigma$ and into $\rho \rho$ are very strong. Also for the $f_{0}(1500)$ we find that the $4 \pi$ decays are important, they cover about half of all decays. The WA102 collaboration reports that the $f_{0}(1500)$ decays into $\rho \rho$ and $\sigma \sigma$ while the $f_{0}(1370)$ decays dominantly into $\rho \rho[30]$. This is incompatible with our findings: the peak in the $4 \pi^{0}$ invariant mass of Fig. 7 and 8 cannot possibly be explained by the $f_{0}(1500)$ alone. The implications of this discrepancy for the scalar states were discussed in [33].

The results quoted above differ from those obtained in [13]. In [13] the $\bar{p} p \rightarrow 5 \pi^{0}$ channel was analyzed without the knowledge from $5 \pi$ final states involving charged particles about the scalar states and the $\pi(1300)$. The main result of that analysis was the need for the $f_{0}(1500)$. It was found to decay strongly into $\sigma \sigma$, but also in $\pi(1300) \pi$ with the $\pi(1300)$ decaying into $\sigma \pi$. In this new analysis it is shown that the $\pi(1300)$ does not decay into $\sigma \pi$. In [13] two solutions were found; one with a broad non-resonant $\sigma \sigma$ interaction, and one with a "narrow" $f_{0}(1370)$. Only the solution with the "narrow" $f_{0}(1370)$ is compatible with the reaction $\bar{p} n \rightarrow \pi^{-} 4 \pi^{0}$. The advantage of the latter reaction is the uniquenes of the $4 \pi^{0}$ system while there are five $4 \pi^{0}$ combinations in the reaction $\bar{p} p \rightarrow 5 \pi^{0}$, and in the construction of $2 \pi^{0}$ and $3 \pi^{0}$ systems the number of combinatorical solutions increases further. Furthermore, the amplitudes for $f_{0} \pi^{0} \rightarrow \sigma \sigma \pi^{0}, f_{0} \pi^{0} \rightarrow \pi(1300) \pi^{0} \pi^{0} \rightarrow$ $\sigma \pi^{0} 2 \pi^{0} \rightarrow 5 \pi^{0}$, and $\pi(1300) \sigma \rightarrow \sigma \pi^{0} 2 \pi^{0} \rightarrow 5 \pi^{0}$ have all the same angular distribution and are difficult to disentangle. These circumstances led to a fit in which the $\pi(1300) \rightarrow \sigma \pi$ decays seem to play a significant role. We note that the likelihood of the fit to the $5 \pi^{0}$ data improves when amplitudes including the $\pi(1300) \rightarrow \sigma \pi$ decay mode e.g. the $f_{0}(1500) \rightarrow \pi(1300)[\rightarrow \sigma \pi] \pi$ decay are introduced. Now, this decay mode is definitely excluded by the new information. This new analysis describes different datasets in a consistent way (see also [26]). The number of events assigned to the $f_{0}(1500)$ and hence the $4 \pi / 2 \pi$ decay ratio of the $f_{0}(1500)$ is reduced compared to [13].

The branching ratios of Table 3 may provide for a discrimination between different glueball-quarkonia mixing scenarios. Decays into two pseudoscalars alone does not seem to allow a decision between different mixing schemes $[2,4]$. Even a decay analysis of the $f_{0}(1500)$ into two pseudoscalar mesons within an instanton driven decay process is compatible with the measured $f_{0}(1500)$ decays into two pseudoscalar mesons [31]. In a recent calculation, decays of the $\bar{q} q$ component of the $f_{0}$ wave functions are calculated using the ${ }^{3} P_{0}$-model; glueball components are supposed to consist of two constituent gluons which convert perturbatively into two $\bar{q} q$ pairs [32]. The calculation is carried out for the mixing schemes of $[2,4]$; the theoretical results do not agree with our measured partial widths (Table 3 ) for neither mixing scheme. So either the approximations made in the model are not valid or the mixing schemes do not correspond to the physical situation. A better understanding of the scalar mesons would require that the decay modes of the three states - $f_{0}(1370), f_{0}(1500), f_{0}(1770)$ - are calculated; this analysis and earlier Crystal Barrel data provide the experimental information at least for the two lower mass states.

\section{Summary}

We have performed a partial wave analysis of the reaction

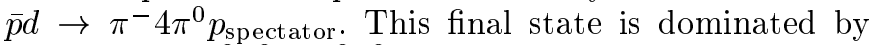
$f_{0}(1370) \rightarrow\left(\pi^{0} \pi^{0}\right)_{s}\left(\pi^{0} \pi^{0}\right)_{s}$. We have determined its mass and width to be $m\left(f_{0}(1370)\right)=1395 \pm 40 \mathrm{MeV} / \mathrm{c}^{2}$ and $\Gamma\left(f_{0}(1370)\right)=275 \pm 55 \mathrm{MeV} / \mathrm{c}^{2}$. We find that $f_{0}(1370)$ decays dominantely into $4 \pi$. Its decay into $\sigma \sigma \rightarrow 4 \pi^{0}$ is more than 4 times larger than into $\pi^{0} \pi^{0}$. The $4 \pi^{0}$ decay of the $f_{0}(1500)$ is about $1 / 3$ of its $2 \pi^{0}$ decay. Including all 


\begin{tabular}{|c||c|c|c|c||c||}
\hline \hline & $\sigma \sigma$ & $\rho \rho$ & $\pi^{*} \pi$ & $a_{1} \pi$ & $4 \pi$ \\
\hline \hline$f_{0}(1370)$ & $106.8 \pm 28.65$ & $55.1 \pm 21.1$ & $36.8 \pm 17.0$ & $12.5 \pm 5.4$ & 211.2 \\
$f_{0}(1500)$ & $10.4 \pm 6.1$ & $5.0 \pm 4.3$ & $19.8 \pm 14.9$ & $4.8 \pm 3.3$ & 39.9 \\
\hline \hline & $\pi \pi$ & $\eta \eta$ & $\eta \eta^{\prime}$ & $K K$ & $\sum$ \\
\hline \hline$f_{0}(1370)$ & $19.2 \pm 7.2$ & $0.4 \pm 0.2$ & & $7.0 \pm 1.6-18.8 \pm 4.0$ & 32.5 \\
$f_{0}(1500)$ & $24.6 \pm 2.7$ & $1.91 \pm 0.24$ & $1.61 \pm 0.06$ & $4.52 \pm 0.36$ & 32.6 \\
\hline
\end{tabular}

Table 3. Product $4 \pi$-branching ratios for $f_{0}(1370)$ and $f_{0}(1500)$ production and decay $\left(\operatorname{BR}\left(\bar{p} p \rightarrow f_{0} \pi \rightarrow A B \pi \rightarrow 4 \pi \pi\right)\right)$ in comparison with the branching ratios into two pseudoscalar states (in units of $10^{-4}$ ). The branching ratios include interferences within the $f_{0}$-decays but exclude interferences with the recoiling pion. The $\rho \rho, \pi^{*} \pi$ and $a_{1} \pi$ branching ratios have been determined in [26] where the $\pi^{*}$ - and $a_{1}$-decay into $\rho \pi$ can be observed. The branching ratios in the second row are taken from [11, $28,29]$.

$4 \pi$ decay modes the $4 \pi$ decay represents about half of its total width.

The $\pi(1300)$ is seen recoiling against two pions in $S$-wave. We find a mass and width of $m(\pi(1300))=1375 \pm 40 \mathrm{MeV} /$ and $\Gamma(\pi(1300))=268 \pm 50 \mathrm{MeV} / \mathrm{c}^{2}$, respectively. Its $(\pi \pi)_{s} \pi$ partial decay widths is smaller than $15 \%$ of its $\rho \pi$ decay width.

We would like to thank the technical staffs of the LEAR machine group and of all the participating institutions for their invaluable contributions to the success of the experiment. We acknowledge financial support from the German Bundesministerium für Bildung, Wissenschaft, Forschung und Technologie, the Schweizerischer Nationalfonds, the British Particle Physics and Astronomy Research Council, the U.S. Department of Energy (contract No.'s DE-FG0387ER40323, DE-AC03-76SF00098 and DE-FG02-

87ER40315) and the National Science Research Fund Committee of Hungary (contract No. OTKA F014357). F.H. Heinsius and K. M. Crowe acknowledge support from the A. von Humboldt Foundation.

\section{References}

1. C. Morningstar and M. Peardon, Phys. Rev. D60 (1999) 34509 .

2. C. Amsler and F. Close, Phys. Rev. Lett. B353 (1995) 385; Phys. Rev. D53 (1996) 295.

3. F. E. Close, G. R. Farrar and Z. Li, Phys. Rev. D55 (1997) 5749 .

4. J. Sexton, A. Vaccarino and D. Weingarten, Phys. Rev. Lett. 75 (1995) 4563.

5. A. V. Anisovich, V. V. Anisovich and A. V. Sarantsev. Z. Phys. A359 (1997) 173.

6. Crystal Barrel Collaboration, V. V. Anisovich et al., Phys. Lett. B323 (1994) 233.

7. Crystal Barrel Collaboration, C. Amsler et al., Phys. Lett. B340 (1994) 259.

8. Crystal Barrel Collaboration, C. Amsler et al., Phys. Lett. B342 (1995) 433.

9. Crystal Barrel Collaboration, C. Amsler et al., Phys. Lett. B353 (1995) 571.
10. Crystal Barrel Collaboration, C. Amsler et al., Phys. Lett. B355 (1995) 425.

11. Crystal Barrel Collaboration, A. Abele et al., Phys. Lett. B385 (1996) 425.

12. The Particle Data Group, C. Caso et al., The European Physical Journal C3 (1998) 1.

13. Crystal Barrel Collaboration, C. Amsler et al., Phys. Lett. B380 (1996) 453.

14. Crystal Barrel Collaboration, E. Aker et al., Nucl. Instrum. Meth. A321 (1992) 69.

15. Crystal Barrel Collaboration, A. Abele et al., Phys. Lett. B391 (1997) 191.

16. D. Herndon, P. Söding and R. J. Cashmore, Phys. Rev. D11 (1975) 3165.

17. S. U. Chung et al., Ann. d. Physik 4 (1995) 404.

18. M. Jacobs and G. C. Wick, Ann. Phys. 7 (1959) 404; C. Amsler and J. C. Bizot, Comp. Phys. Comm. 30 (1983) 21.

19. C. J. Batty, Nucl. Phys. A601 (1996) 425.

20. Preliminary results of an analysis of $\bar{p} n \rightarrow K^{-} K_{s} \pi^{0}$ (Crystal Barrel Collaboration).

21. L. Rosselet et al., Phys. Rev. D15(1977) 574.

22. G. Grayer et al., Nucl. Phys. B75 (1974) 189.

23. M. Gaspero, Nucl. Phys. A562 (1993) 407.

24. A. Adamo et al., Nucl. Phys. A558 (1993) 13c.

25. Crystal Barrel Collaboration, C. Amsler et al., Phys. Lett. B322 (1994) 431.

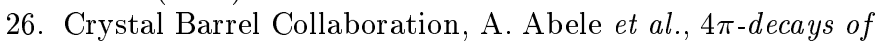
scalar and vector mesons, in preparation.

27. D. V. Bugg et al., Phys. Lett. B353 (1995) 378.

28. A. Abele et al., Nucl. Phys. A609 (1996) 562.

29. Crystal Barrel Collaboration, A. Abele et al., Phys. Rev. D57 (1998) 3860.

30. WA102 Collaboration, D. Barberis et al., Phys. Lett. B471 (2000) 440.

31. C. Ritter et al. Phys. Lett. B380 (1996) 431.

32. M. Strohmeier-Presicek et al., Phys. Rev. D60 (1999) 54010 .

33. E. Klempt, Meson'2000 workshop, Cracow, Poland, May 2000 ,

On the existence of glueballs and hybrids 